\title{
L'illusion de la verve surréaliste dans Capitale de la douleur
}

Pascale Roux

\section{(2) OpenEdition}

1 Journals

Édition électronique

URL : https://journals.openedition.org/recherchestravaux/708

DOI : 10.4000/recherchestravaux.708

ISSN : 1969-6434

Éditeur

UGA Éditions/Université Grenoble Alpes

\section{Édition imprimée}

Date de publication : 15 décembre 2014

Pagination : 47-60

ISBN : 978-2-84310-291-2

ISSN : 0151-1874

Référence électronique

Pascale Roux, «L'illusion de la verve surréaliste dans Capitale de la douleur », Recherches \& Travaux [En ligne], 85 | 2014, mis en ligne le 15 juin 2016, consulté le 28 juin 2022. URL : http://

journals.openedition.org/recherchestravaux/708; DOI : https://doi.org/10.4000/recherchestravaux. 708

(c) Recherches \& Travaux 


\section{L'illusion de la verve surréaliste dans Capitale de la douleur}

"J'ai la beauté facile et c'est heureux $x^{\mathrm{I}}$. Ce vers de Capitale de la douleur trace les contours de ce qui pourrait être une définition de la verve éluardienne. Dans cette prosopopée de la parole, l'auteur met en scène un rapport à la langue poétique marqué par l'aisance et la créativité. Un rapport facile : spontané et léger, comme l'est une femme facile, qui se prête de bonne grâce à tous les désirs. Un rapport heureux : sans souffrance et réussi, comme l'est une trouvaille heureuse, source de créativité et de poéticité.

Que le terme soit employé dans un contexte évaluatif positif ou négatif, parler de «verve» implique que l'on prenne en compte l'acte d'énonciation, et plus précisément que l'on s'interroge sur la manière dont sont mis en scène le moment et les modalités d'émergence de la parole. La notion, fréquemment utilisée dans le domaine oratoire, l'est plus rarement dans le domaine littéraire, où la production écrite est coupée de sa situation matérielle de profération. Pour la mobiliser dans le cas d'un texte littéraire, il faut que, d'une manière ou d'une autre, ce rapport facile et heureux à la langue et à la beauté poétique fasse l'objet d'une mise en scène textuelle.

La verve littéraire nous paraît ainsi se définir comme une posture énonciative, l'une des formes que peut prendre l'éthos de l'écrivain. Mais, comme toute mise en scène énonciative, elle comporte d'une part des signaux textuels et d'autre part une visée, ou au moins un effet : elle programme ou provoque une réception et une interprétation du discours. Le lecteur qui identifie les signaux de la verve adopte une certaine attitude face au texte, que l'on peut

I. «La Parole», dans Capitale de la douleur [1926], Paris, Gallimard, 20I2, p. 2I. Dans la suite de l'article, les renvois aux pages entre parenthèses correspondent à cette édition. 
schématiquement décrire comme symétrique à l'attitude de l'énonciateur : il se laisse porter par une parole aisée et créative, il en tire plaisir parce qu'elle se donne comme telle. Ainsi, la verve nous semble participer d'une forme de rhétorique : elle se manifeste par une série de procédés, dont on peut penser qu'ils se retrouvent dans différents textes et chez différents auteurs, et elle met en jeu une relation énonciative, puisqu'elle présuppose une posture de l'énonciateur et qu'elle programme une réception.

Capitale de la douleur recourt aux codes d'une verve surréaliste², qui résulte non de l'inspiration mais d'une expérience, décrite par André Breton au sujet des Champs magnétiques:

Dans l'ensemble, [les résultats] de Soupault et les miens présentaient une remarquable analogie : même vice de construction, défaillance de même nature, mais aussi, de part et d'autre, l'illusion d'une verve extraordinaire, beaucoup d'émotion, un choix considérable d'images d'une qualité telle que nous n'eussions pas été capables d'en préparer une seule de longue main, un pittoresque très spécial et, de-ci de-là, quelque proposition d'une bouffonnerie aiguë.

La verve est une «illusion», dit Breton : elle n'est pas liée à un don, à une aptitude ou même à une capacité du poète, mais elle produit un effet similaire.

L'effet de verve tient notamment à la mise en scène de l'émergence spontanée de la parole, ainsi qu'au recours à des marques textuelles. Parmi celles-ci, certaines sont syntaxiques, comme le désordre syntaxique, signal de la spontanéité d'une parole non soumise au contrôle de la raison, ou l'accumulation, signal d'abondance d'une parole profuse. D'autres sont liées aux images, comme la structure de dérive, qui reproduit le flot originel de la parole, ou comme l'incohérence des signifiés, signe, pour les surréalistes, de la fantaisie qui naît de la dictée de l'inconscient. Mais que l'on se situe au niveau de la posture énonciative du poète ou à celui des marques textuelles, Éluard joue des codes de la verve surréaliste, il les détourne, parfois les subvertit ou y recourt pour égarer le lecteur.

\section{Le poète en verve : postures énonciatives}

Le rapport facile de l'énonciateur à la parole poétique est mis en scène en plusieurs occasions dans le recueil, par exemple dans les premiers vers de "L'invention », dont le titre indique, par référence à l'inventio rhétorique, qu'il décrit le processus de la création :

2. Et, pour les poèmes les plus anciens, à ceux d'une verve dadaïste.

3. A. Breton, Manifestes du surréalisme [1924, I930, 1942], Paris, Gallimard, coll. "Essais», 2000, p. 34. Je souligne. 
La droite laisse couler du sable.

Toutes les transformations sont possibles.

Le sable est ici une métaphore de la parole poétique, qui s'écoule comme d'ellemême et échappe à la maîtrise de l'écrivain, représenté par synecdoque par sa main, «la droite». Et c'est l'écoulement spontané de la parole qui ouvre le champ des possibles poétiques, qui lui donne son pouvoir de transformation et de transfiguration.

Les métaphores d'une parole échappant au contrôle du poète, et par là même créative, sont nombreuses : le sable, mais aussi le vent ou l'eau ${ }^{4}$, ou encore l'ivresses. La posture du poète en verve est ici définie par le renoncement de celui-ci à exercer un contrôle sur la parole, mise en scène dans la spontanéité de son engendrement. Cette posture implique une forme de passivité et d'effacement du je parlant, dont on trouve quelques expressions frappantes. Le poème en prose «Nul» (p. 24), par exemple, s'ouvre ainsi : "Ce qui se dit : [...]». Que l'on donne à la forme pronominale une interprétation passive (ce qui est dit) ou autocausative (ce qui se dit tout seul), dire est ici un procès dont l'actant sujet est effacé. Le motif est repris à la fin du poème, dont la dernière séquence commence, de manière symétrique, par l'expression "À son tour un cri : [...]» : le procès, présenté sous une forme nominale déverbale, l'est encore indépendamment de l'actant sujet. On a ici une représentation hyperbolique d'une forme de verve poétique : la parole est mise en scène dans la spontanéité absolue de son émergence, comme si elle se déployait d'ellemême, indépendamment de tout énonciateur.

On perçoit le lien que cette posture de l'énonciateur en verve peut entretenir avec l'esthétique surréaliste. L'automatisme postule en effet, à différents degrés et de différentes manières, que la spontanéité de la production poétique, non soumise à la censure de la raison et de la morale, est au fondement de la créativité poétique. La verve surréaliste se met ainsi en scène dans des situations archétypiques de production, comme le sommeil ou le rêve, censées favoriser un rapport à la langue immédiat, fécond en termes poétiques. Ces motifs sont largement repris et exploités dans Capitale de la douleur, et ils fonctionnent parfois très clairement comme des éléments de mise en scène de l'énonciation. La composition de "Première du monde" (p. 97-98), par exemple, le montre. Dans les deux quatrains d'alexandrins qui encadrent le poème se trouvent des images du sommeil : il s'agit du cadre de la parole poétique, cadre

4. Comme dans «La Parole» ("Je glisse sur le toit des vents / Je glisse sur le toit des mers», p. 2I) ou encore dans «La Rivière» ("La rivière que j'ai sous la langue, / L'eau qu'on n'imagine pas, mon petit bateau", p. 22).

5. Dans «Boire» (p. IO4), par exemple. 
à la fois structurel et énonciatif. Les trois quatrains d'octosyllabes centraux, où se développent une série d'images typiquement surréalistes, peuvent être interprétés comme décrivant le rêve de la dormeuse évoquée dans les strophes encadrantes $^{6}$; mais ils constituent aussi la parole poétique d'un énonciateur dont les strophes encadrantes fondent l'éthos. Le sujet s'efface derrière une parole qui tout à la fois l'oblitère et le porte, même si, puisque cet effacement est mis en scène textuellement, il s'agit encore d'une posture d'énonciation et d'une forme de rhétorique : il postule un événement initial à l'origine de l'énoncé, cet événement constituant en même temps la caution de celui-ci'.

Malgré cet effacement de l'énonciateur, Éluard, dans Capitale de la douleur, est aussi poète du je; si l'on veut parler de sa verve surréaliste, il faut donc préciser qu'elle est également lyrique. Ce lyrisme entre dans une certaine mesure en contradiction avec sa verve surréaliste, puisque l'une et l'autre présupposent des postures du sujet opposées : alors que l'une est fondée sur son effacement, l'autre l'est sur sa représentation, éventuellement hyperbolique, et son expression. L'art poétique exposé dans «La Parole» fait apparaître cette contradiction :

Je suis devenue sentimentale

Je ne connais plus le conducteur

La parole est lyrique, «sentimentale», mais elle se déploie indépendamment du sujet, «le conducteur». La verve d'Éluard est ainsi marquée par la position paradoxale du sujet : son lyrisme est travaillé par un rapport surréaliste à la parole et, inversement, sa posture surréaliste est travaillée par le lyrisme. Deux autres vers de «La Parole» font apparaître cette double posture :

J'aime le plus chinois aux nues

J'aime la plus nue aux écarts d'oiseau

Si la fonction expressive de la langue fonde la parole poétique («j’aime»), celle-ci est caractérisée par son opacité, mise en abyme par l'expression «le plus chinois ", et par l'autonomie de son fonctionnement : les signifiants s'engendrent les uns les autres, selon un principe phonétique ${ }^{8}$ et non sémantique.

6. Le poème se trouve en annexe du présent article.

7. Voir sur ce point L. Jenny : «L'écriture automatique, aussi spontanée qu’elle se veuille en son départ, n'échappe pas à une certaine existence rhétorique, au double sens où une rhétorique programme la production mais aussi la réception d'événements de discours. [...] En deçà des formes réelles, on doit effectivement postuler un événement initial, à la fois délibéré et involontaire, qui arrache le discours à la transparence et lui donne toute sa valeur.» («Les aventures de l'automatisme», Littérature, $\mathrm{n}^{\circ} 72$, 1988, p. 3.)

8. Les vers s'engendrent sur un principe phonétique et non sémantique, comme en témoigne la reprise des séquences phoniques [ny], dans «nues» et «nue», et [wazo], dans "chinois aux» et «oiseau». 
La verve éluardienne est ainsi simultanément surréaliste et lyrique, elle postule à la fois l'effacement du sujet et son affirmation. Mais, quoi qu'il en soit, il s'agit d'une posture et, en cela, la verve poétique y est une «illusion", selon le mot de Breton. Elle est également un effet du texte qui, par des procédés stylistiques, mime la spontanéité d'engendrement de la parole poétique - la «beauté facile». On relèvera, parmi les signaux textuels de la verve, deux séries : au niveau syntaxique, le phénomène du rejet de l'ordre grammatical et celui de l'accumulation; au niveau des images, le principe de la dérive.

\section{L'effet de verve : la syntaxe et les images}

Dans la rhétorique automatique, l'effet de verve est produit notamment par une langue qui désorganise la syntaxe et, ainsi, fait signe vers la spontanéité et le désordre de la parole automatique - le «vice de construction" que Breton constatait lorsqu'il commentait Les Champs magnétiques.

La poésie d'Éluard bouleverse peu la syntaxe, sauf parfois dans la première partie du recueil, c'est-à-dire dans les poèmes les plus anciens; mais, souvent marquée par la parataxe et l'asyndète, sa langue réduit au minimum la fonction d'organisation et de hiérarchisation de la syntaxe. Cette langue, figurant la spontanéité d'une parole qui émerge sans l'intervention régulatrice du sujet, permet, outre l'effet de verve qu'elle produit, que se démultiplient les sens possibles. Elle est ainsi féconde, en termes poétiques. Les effets de la parataxe et de l'asyndète sont variés en contexte, mais on peut au moins signaler que cette organisation est analogue au procédé du collage surréaliste : des mots ou des séquences sont juxtaposés, non reliés par des relations explicites mais inclus dans un cadre commun, la phrase et le poème, incitant l'interprétation à tisser des liens entre eux.

La langue de Capitale de la douleur recourt par ailleurs fréquemment à l'ambiguïté syntaxique, et notamment à l'amphibologie", qui introduit du désordre, puisqu'elle perturbe l'identification des relations organisant les constituants, rendant plus difficile l'accès au sens de l'énoncé. On observe ce phénomène par exemple dans le poème intitulé «Suite» (p. 20) :

Pour l'éclat du jour des bonheurs en l'air

Pour vivre aisément des goûts des couleurs

Pour se régaler des amours pour rire

Pour ouvrir les yeux au dernier instant

Elle a toutes les complaisances.

9. Construction grammaticale ambiguë entraînant une ambiguïté sémantique. 
Le parallélisme syntaxique, associé ici à l'accumulation, peut, formellement, fonctionner comme un principe organisateur de la phrase, puisqu'il permet d'ordonner celle-ci selon un principe de répétition. Mais il s'agit aussi d'un principe de relance automatique ${ }^{10}$ caractéristique de la verve surréaliste. Par ailleurs, le parallélisme complique la lecture syntagmatique d'une lecture paradigmatique : il a pour effet de démultiplier les liens qu'il est possible de tisser, sur le plan sémantique, entre les constituants, et de démultiplier les possibles interprétatifs. La lecture de ce poème est rendue encore plus complexe par l'amphibologie. Les sens sont multiples car les relations syntaxiques sont équivoques : les trois premiers vers peuvent, grammaticalement, se lire soit comme une suite de deux syntagmes indépendants, formant une phrase elliptique ou non verbale, soit comme un seul syntagme incluant un complément, sur le modèle du quatrième vers. L'ordre syntaxique est perturbé, il ne permet pas d'ordonner clairement et de manière univoque les mots les uns par rapport aux autres et il est concurrencé, dans sa fonction organisatrice, par un système de relations verticales.

L'accumulation marque quant à elle la profusion de la parole poétique, et ce malgré le fait que les poèmes sont généralement brefs, parfois réduits à quelques vers ou lignes. Même dans le cas des textes marqués par leur clôture, l'accumulation fait signe vers le flot de la parole poétique, virtuellement ouvert. On le constate par exemple dans ce court poème en prose, qui est l'un des "textes automatiques» du recueil ${ }^{\text {II }}$ :

Grandes conspiratrices, routes sans destinées, croisant l'x de mes pas hésitants, nattes gonflées de pierres ou de neige, puits légers dans l'espace, rayons de la roue des voyages, routes de brises et d'orages, routes viriles dans les champs humides, routes féminines dans les villes, ficelles d'une toupie folle, l'homme, à vous fréquenter, perd son chemin et cette vertu qui le condamne aux buts. Il dénoue sa présence, il abdique son image et rêve que les étoiles vont se guider sur lui.

Que l'interprétation s'appuie, pour identifier le destinataire du poème, sur la reprise d'un topos amoureux - lecture favorisée par la surreprésentation du féminin linguistique dans le texte - ou sur celle du motif surréaliste de l'errance, le lecteur est pris dans le flot d'une parole qui multiplie les prédicats associés au "vous", par une relation attributive sous-jacente. Cette accumulation n'est ni une énumération, close ou virtuellement close, ni une gradation ordonnée, mais une série ouverte qui ne se donne pas comme organisée. La démultiplication des prédicats, redoublée par le fait que les noms en tête de

Io. Au sujet de la relance automatique, voir Manifestes du surréalisme, ouvr. cité, p. 4I-42.

II. J.-C. Gateau, "Éluard et le texte surréaliste», Une pelle au vent dans les sables du rêve, Lyon, Presses universitaires de Lyon, 1992, p. 65-72. 
chaque syntagme figurent tous au pluriel, met en abyme la multiplicité des possibles qui s'offrent à la parole poétique, comme ces innombrables routes qui s'ouvrent devant le marcheur sans destinée. Juxtaposition et accumulation produisent ici un effet de verve car elles suggèrent l'ouverture à d'autres possibles poétiques et signalent la profusion de la parole, mais aussi car elles multiplient les possibles interprétatifs, les constituants internes de chacun des groupes entrant en correspondance les uns avec les autres. La langue poétique est ainsi à la fois profuse et féconde, elle est féconde parce que profuse et désordonnée.

Mais ce procédé caractéristique de la verve surréaliste, ce code de la rhétorique automatique, est ici contourné : il entre en tension avec une autre structure, celle de la période ${ }^{\mathrm{r}}$. Le texte est en effet construit selon le schéma suivant : une protase (l'accumulation), une acmé («l'homme»), une apodose (la fin de la phrase); la phrase suivante peut s'analyser et s'oraliser comme une clausule, fournissant la conclusion de ce qui précède. Cette structure, qui incite à une lecture oratoire du texte, produit elle aussi un effet de verve, mais elle entre en tension avec la rhétorique automatique, d'abord parce qu'il s'agit d'une structure d'énoncé et d'un ordre syntaxique classiques, a priori incompatibles avec la mise en scène énonciative automatique. Par ailleurs, l'éclatement caractéristique de l'écriture automatique, identifié par le lecteur dans la première partie du texte, est annulé par la reprise anaphorique au moyen de "vous», qui redonne une forte cohérence à l'énoncé, à la fois sur le plan syntaxique (a posteriori, tous les groupes nominaux sont analysés comme des appositions), sur le plan énonciatif (ils ont tous une fonction de caractérisation du destinataire) et sur le plan thématique (la structure binaire de l'énoncé : le féminin dans la protase, le masculin à partir de l'acmé).

L'accumulation, tout comme la parataxe, l'asyndète et les ambiguïtés syntaxiques, désignent le moment originel de l'émergence de la parole automatique, spontanée, non réglée par un sujet l'organisant de manière claire, explicite et univoque. L'accumulation marque non seulement la profusion de la parole, mais aussi, d'une certaine manière, le refus de la sélection par l'énonciateur, qui se met en scène n'opérant pas de choix dans la série des images qui se présentent à lui. La verve d'Éluard met ainsi en scène le rejet de l'intervention régulatrice de la raison, même si ce rejet est bien moins marqué que dans d'autres textes surréalistes.

Dans la rhétorique surréaliste, la dérive des images, au sens large que le premier Manifeste donne à ce dernier terme, figure stylistiquement une parole qui se donne non pas comme structurée en amont par l'énonciateur, voulue

I2. Nous remercions F. Rouffiat pour sa lecture éclairante de ce poème. 
et pensée comme poétique, résultant d'un travail sur un matériau linguistique résistant, mais comme portant le sujet, pris dans le flot de son propre dire. Michaël Riffaterre a analysé ce phénomène de la dérive des images surréalistes $^{13}$ : celles-ci, obscures et déconcertantes si on les considère isolément, sont rattachées les unes aux autres par «une chaîne ininterrompue d'associations verbales». Le facteur contrôlant le déroulement de la chaîne métaphorique est un principe d'association formelle : un mot de la séquence détermine l'occurrence des mots qui le suivent, en vertu soit d'une similitude de forme (par exemple le parallélisme phonétique, les jeux de mots), soit d'associations stéréotypées (par exemple le cliché ou la citation). La parole poétique est ainsi montrée dans la spontanéité de son engendrement, et ce fonctionnement est au cœur de la créativité poétique surréaliste : la logique associative formelle permet que soient associés "des signifiants dont les signifiés sont incompatibles", et que soit ainsi renouvelée la vision monde. Selon les mots de Riffaterre, «la représentation de la réalité en est bouleversée» ou, selon les mots d'Éluard cités plus haut, "toutes les transformations sont possibles».

Éluard reprend ce principe de la verve surréaliste automatique, et parfois il en joue, lorsqu'il désorganise et en brise la chaîne associative, par exemple en inversant l'ordre des éléments qui la composent. C'est le cas dans le poème «Une» (p. II7), traversé par l'association stéréotypée de la femme et de l'étoile et par l'intertexte nervalien, qui se clôt ainsi : «Blanche éteinte des souvenirs, étalée, étoilée, rayonnante de tes larmes qui fuient. Je suis perdu.» Au centre se trouvent deux termes phonétiquement proches : «étoilée » est cohérent avec le contexte antérieur, "étalée " apparaît par variation formelle. Mais la chaîne associative a été brisée, le terme cohérent contextuellement n'apparaissant qu'après celui qui, pourtant, semble en dériver. Le principe est le même pour les deux groupes adjectivaux encadrants : «rayonnante de tes larmes» ne pose pas de problème majeur d'interprétation, car il est cohérent avec le contexte antérieur et syntaxiquement correct; " éteinte des souvenirs", par contre, n'est pas syntaxiquement correct et est manifestement formé par calque sur l'autre groupe adjectival qui, pourtant, n'apparaît qu’à la fin de la séquence. Éluard exploite le principe d'association formelle de l'automatisme, mais il en désorganise la chaîne, ce qui a pour effet de rendre la parole poétique plus résistante à l'interprétation : non seulement les signifiés associés par les images génèrent des représentations étranges, mais la logique de leur association est brouillée. La phrase finale, "Je suis perdu», a ainsi, outre son interprétation lyrique, une portée métalinguistique : la chaîne associative des signifiants, rompue, finit

I3. M. Riffaterre, «La métaphore filée dans la poésie surréaliste ", Langue française, nº 3, I969, p. $46-60$. 
par perdre aussi bien l'énonciateur que le lecteur. Le principe de la spontanéité d'engendrement de la parole poétique entre ici en tension avec le travail de réorganisation du poète.

Le second poème intitulé «À la flamme des fouets» (p. IO2), fonctionne également par dérive interne des images, notamment la première série de quatre vers, dont Agnès Fontvieille-Cordani a analysé la logique de progression, formelle et sémantique ${ }^{\mathrm{T} 4}$ :

Métal qui nuit, métal de jour, étoile au nid,

Pointe à frayeur, fruit en guenilles, amour rapace,

Porte-couteau, souillure vaine, lampe inondée,

Souhaits d'amour, fruits de dégoût, glaces prostituées.

Mais, dans ce poème, la dérive des images n'est pas uniquement interne au texte, elle est aussi externe : on remarque notamment que, du terme "corset», que l'on trouve dans le premier poème intitulé «À la flamme des fouets» (p. IOI), dérivent ces deux vers du second poème :

Bien sûr, bonjour à vos harpons,

À vos cris, à vos bonds, à votre ventre qui se cache!

L'expression "votre ventre qui se cache" développe l'un des sens de "corset", et le terme "harpons" apparaît dans une chaîne d'associations stéréotypées : corset $\rightarrow$ baleines $\rightarrow$ harpons. La chaîne des associations est ici brouillée dans sa linéarité, en raison de l'éloignement des termes qui la composent et de leur appartenance à deux ensembles textuels distincts.

La verve éluardienne, surréaliste en ce qu'elle procède d'une chaîne d'association des signifiants, a ainsi ceci de spécifique qu'elle montre et cache tout à la fois le principe d'émergence spontané de la parole poétique. Le plaisir du lecteur, dès lors, est de reconstituer la chaîne associative originelle et d'apprécier les effets liés à sa déstructuration, de saisir la manière dont le matériau linguistique premier a fait l'objet d'un travail de désorganisation et de réorganisation.

Dans Capitale de la douleur, Éluard exploite les principes de l'effet de verve surréaliste tout en les déjouant, ou plus précisément en faisant se déployer sa langue poétique au point de tension entre l'ordre (celui de la syntaxe ou de la logique associative à l'œuvre dans la série des images) et le désordre. La posture de l'énonciateur est ainsi à la fois celle du poète en verve - profusion et spontanéité - et celle du poète travaillant le matériau linguistique de la parole spontanée.

I4. A. Fontvieille-Cordani, Paul Éluard: l'inquiétude des formes, Lyon, Presses universitaires de Lyon, 20I3, p. Ioo. 


\section{La verve et l'énigme : incohérence et cohérence des signifiés}

En vertu du principe de la dérive, l'image surréaliste repose sur une cohérence des signifiants, et son étrangeté est produite par l'incohérence des signifiés associés, au niveau macrostructurel (l'incohérence de l'ensemble des images du texte) ou microstructurel (celle des signifiés associés dans un cadre syntaxique donné). La définition que Breton donne de l'image dans le premier Manifeste $e^{15}$, reprenant et radicalisant celle de Reverdy, est tout entière fondée sur les effets de dépaysement produits par cette incohérence. Capitale de la douleur joue parfois avec ce code. En effet, dans certains poèmes, l'incohérence des signifiés, que le lecteur a tendance à interpréter comme une marque d'automatisme, n'est qu'apparente.

C'est le cas par exemple dans «Le plus jeune» (p. II8), qui commence ainsi :

Au plafond de la libellule

Un enfant fou s'est pendu,

Fixement regarde l'herbe,

Confiant lève les yeux

La métaphore par complément déterminatif qui ouvre le poème est fondée sur l'appariement syntaxique de deux termes incompatibles sur le plan sémantique, que le lecteur ne parvient pas à articuler l'un à l'autre : le transfert métaphorique est bloqué et la relation établie par de est difficile à déterminer. On a ici un cas assez caractéristique de "collage référentie ${ }^{16}$ » : le lecteur se trouve dans l'incapacité d'identifier le référent désigné par l'expression, et un effet d'étrangeté découle de l'incohérence des signifiés. Mais celle-ci disparaît une fois l'énigme du texte élucidée : le poème décrit un enfant pendu par les pieds, voyant le monde à l'envers, représentation que l'on trouve sur l'arcane XII du jeu de tarot de Marseille. Le "plafond de la libellule» désigne métaphoriquement la branche de l'arbre à laquelle l'enfant est suspendu, présente dans certaines représentations de l'arcane. On note par ailleurs que ces vers favorisent une interprétation s'appuyant sur une représentation stéréotypée de la mort, notamment en raison du passage de "fixement» à "confiant" et du mouvement des yeux, interprété spontanément comme allant du bas vers le haut, ce qui ouvre à une interprétation religieuse (la confiance comme voie de la résurrection). L'ambiguïté de la forme "se pendre», que l'on peut interpréter soit comme un verbe pronominal autonome (signifiant alors «se

I5. A. Breton, Manifestes du surréalisme, ouvr. cité.

I6. Nous empruntons l'expression à M. Murat, qui l'a utilisée notamment dans «Corps et biens ou les beaux effets du surréel ", L'Information grammaticale, n 25 , 1985, p. 36-40. 
donner la mort par pendaison»), soit comme une construction réfléchie, avec un pronom complément (signifiant «suspendre») oriente le lecteur sur une fausse piste interprétative. Les deux vers suivants jouent sur les incertitudes interprétatives que peut provoquer l'asyndète, qui n'explicite pas le lien entre les propositions juxtaposées : le lecteur identifie spontanément un lien de succession (puis), présupposant que l'herbe se trouve aux pieds de l'enfant, alors qu'il s'agit d'un lien de simultanéité, les deux propositions désignant en réalité une même direction du regard.

L'un des ressorts du fonctionnement des poèmes-énigmes du recueil est ce type de subversion de l'image surréaliste : les signifiés associés sont en apparence incohérents mais, une fois l'énigme résolue, s'ordonnent autour d'un référent unique, redonnant au texte une forte cohérence. C'est également le cas de «À la flamme des fouets I» (p. IO2), éloge de Sade difficile à identifier à la première lecture, malgré la référence assez nette à celui-ci dans le titre.

Ces beaux murs blancs d'apothéose

Me sont d'une grande utilité.

Tout au sérieux, celui qui ne paie pas les dégâts

Jongle avec ton trousseau, reine des lavandes.

Est-il libre? Sa gorge montre d'un doigt impérieux

Des corridors où glissent les sifflets de ses chevilles.

Son teint, de l'aube au soir, démode ses tatouages

Et l'asile de ses yeux a des portes sans nuages.

Ô régicide! ton corset appartient aux mignons

Et aux mignonnes de toutes sortes. Ta chair simple s'y développe,

Tu t'y pourlèches dans la pourpre, ô nouveau médiateur!

Par les fentes de ton sourire s'envole un animal hurleur

Qui ne jouit que dans les hauteurs.

Le poème évoque la période d'incarcération de Sade, au cours de laquelle il rédigea la plus grande partie de son œuvre, et plus particulièrement, à la fin du poème, un épisode précis de son embastillement ${ }^{17}$. Le texte induit en erreur, d'abord parce que la configuration énonciative change à chacune des strophes : Sade est successivement représenté comme le locuteur ( $j e$ ), le délocuté (il) et l'allocutaire $(t u)$. Il est également égarant en raison des images, qui renvoient indirectement à des éléments de la vie et de l'œuvre de Sade mais sont, en

17. Le 2 juillet 1789, ayant fabriqué un porte-voix, Sade se mit à crier du haut des tours de la Bastille qu'on égorgeait les prisonniers. C'est cet épisode, dont Éluard fait le récit dans des articles qu'il consacre à Sade, que transposent les deux derniers vers du poème : «Par les fentes de ton sourire s'envole un animal hurleur / Qui ne jouit que dans les hauteurs. » Parmi les textes d'Éluard sur Sade, on peut citer : «D. A. F. de Sade, écrivain fantasque et révolutionnaire», La Révolution surréaliste, $\mathrm{n}^{\circ} 8, \mathrm{I}^{\text {er }}$ décembre 1926, p. 8-9; "L'intelligence révolutionnaire : le marquis de Sade (I740-I8I4) ", Clarté, nouvelle série, I927, I5 février, nº 6, p. 30. 
apparence, marquées par une incohérence des signifiés typiquement surréaliste. Dans les deuxième et troisième vers, par exemple, l'évocation indirecte de l'univers carcéral, et notamment de la figure du gardien de prison, désigné par la périphrase ("celui qui ne paie pas les dégâts») et la mention du "trousseau», se clôt par une apostrophe étrange, "reine des lavandes», que l'on pourrait être tenté, dans une première lecture, de ne pas interpréter, en l'associant uniquement à la rhétorique de l'automatisme. Mais son incohérence disparait une fois le référent identifié : la Bastille, qui porte un nom désignant, en provençal, un village fortifié souvent situé sur les hauteurs ${ }^{18}$. On peut également citer, un peu plus loin dans le texte, l'image «l'asile de ses yeux a des portes sans nuages», qui fait référence à la fois à l'incarcération de Sade (en vertu des associations stéréotypées yeux/fenêtres/porte) et au fait que l'auteur a terminé sa vie dans un asile d'aliénés.

Le poème, dans son ensemble, repose sur l'hétérogénéité du réseau de ses signifiés, en particulier de ceux qui ont trait au religieux, au sexuel et au politique. Ces réseaux convergent au début de la troisième séquence. Outre la référence au rôle joué indirectement par Sade dans la chute de la monarchie, la phrase fait se superposer plusieurs réseaux d'images en apparence incohérents mais qui, par leur emboîtement même, figurent la force provocatrice à la fois du personnage et du poème : le Divin Marquis est le «nouveau médiateur» du texte, celui dont le texte mime l' "apothéose», celui qui lui-même disait «Quand l'athéisme voudra des martyrs, qu'il les désigne, et mon sang est tout prêt ${ }^{19}$." L'incohérence des signifiés associés dans ce poème, qu'une lecture inattentive pourrait simplement interpréter comme un effet de verve surréaliste, comme un signal d'une parole spontanée née de la dictée de l'inconscient, est en réalité au fondement même de la puissance poétique, ici subversive.

Ce type de poème joue des codes de la verve surréaliste et les déjoue, en organisant la cohérence des signifiés autour d'un référent caché, selon le principe de l'énigme. Les marques textuelles de la verve sont ici égarantes, dans la mesure où elles mettent en scène la parole dans la spontanéité de son émergence, où elles supposent que l'énonciateur se laisse porter par le flot de son propre dire. L'identification du fonctionnement du poème, énigme résultant d'un travail de codage, d'un jeu sur l'équivoque, transforme l'image du poète. L'énonciateur de l'énigme produit en effet un énoncé obscur et équivoque, adressé à deux destinataires dont les postures s'opposent ${ }^{20}:$ le non-initié, qui

I8. Sade appartenait à une grande famille de Provence.

19. Cette citation est utilisée par Éluard en épigraphe du poème en prose «La Dame de carreau" (La Révolution surréaliste, $\mathrm{n}^{\circ}$ 6, $\mathrm{I}^{\mathrm{er}}$ mars 1926).

20. J.-M. Adam décrit la structure énonciativo-pragmatique de l'énigme en s'appuyant sur un autre poème de Capitale de la douleur (le poème II des « Petits justes»), dans "Le style dans la langue et dans les textes ", Langue française, n' 135, 2002, p. 7I-94. 
n'a pas résolu l'énigme, reste à la surface du texte, n'en percevant ni le sens ni l'enjeu véritable; l'initié, qui l'a résolue, a accès à un second niveau de compréhension et d'interprétation du texte. Les signaux de la verve automatique, qui incitent à ne pas chercher à organiser la parole autour d'un principe unifiant les signifiés et autour de l'identification d'un référent, participent de cette configuration énonciativo-pragmatique de l'énigme. Peut-être peut-on encore parler de "verve» dans ces poèmes, puisque le texte, à la première lecture, en produit les effets, mais en tout cas plus au sens où le terme désigne la posture d'un énonciateur entretenant un rapport immédiat et spontané à la langue. La verve y est un procédé de brouillage du sens de l'énoncé, elle permet à l'énonciateur de désorienter le lecteur, et parfois même de l'égarer.

Ainsi, la verve surréaliste se définit relativement à un événement de parole automatique à l'origine de l'énoncé. Elle correspond à une posture de l'énonciateur parlant sous la dictée de l'inconscient et qui se laisse porter par un dire auquel il n'impose la censure ni de la raison ni de la morale. Elle comporte des marques stylistiques, qui font signe vers cette origine de la parole et qui, générant ce que nous avons appelé un "effet de verve", orientent la réception. La posture de lecture impliquée par la verve surréaliste est, d'une certaine manière, symétrique à celle de l'énonciateur : il s'agit, pour l'un comme pour l'autre, de se laisser porter par la parole, de se laisser dériver, de tirer plaisir des surprises de la langue poétique sans chercher à restaurer un ordre a priori exclu de l'événement de parole originel. La verve incite le lecteur à jouir des trouvailles poétiques, à explorer le champ des possibles interprétatifs ouverts par la démultiplication des liens, sémantiques et syntaxiques, qu'il est possible d'établir à la fois entre les signifiants et les signifiés. La parole ne se ferme pas sur un sens unique mais ouvre sur des sens multiples et inépuisables.

Éluard joue avec les codes de la verve surréaliste, il se les réapproprie en les contournant et, dans un certain nombre de textes, les déjoue. Le lecteur hésite ainsi constamment entre deux postures : celle que les signaux de la verve favorisent - se laisser porter par la parole poétique sans rechercher une interprétation unifiante du texte - et celle que les marques d'un travail de réorganisation de la parole impliquent - rechercher un principe de cohérence textuelle, non pas seulement au niveau du signifiant, mais à celui du signifié et de la référence.

Les poèmes qui fonctionnent comme des énigmes font apparaître de manière plus vive encore cette double posture interprétative impliquée par les textes. Au niveau de l'œuvre dans son ensemble, ces poèmes ont en outre pour effet de renforcer l'hésitation du lecteur concernant sa posture. Puisque le lecteur qui a résolu une énigme fait automatiquement l'hypothèse que d'autres poèmes 
- peut-être tous - fonctionnent de manière analogue, il est tenté de relire tous les poèmes comme potentiellement organisés autour d'un référent caché. Les signaux de la verve automatique sont alors globalement l'objet de la méfiance du lecteur, qui tout à la fois perçoit la mise en scène de l'événement de parole originel à laquelle ils renvoient et la met en doute. Et, à partir de cet événement de lecture, la verve éluardienne devient source à la fois d'inconfort interprétatif et de jouissance poétique, et elle rend le recueil proprement inépuisable.

\section{Première du monde}

À Pablo Picasso

Captive de la plaine, agonisante folle, La lumière sur toi se cache, vois le ciel :

Il a fermé les yeux pour s'en prendre à son rêve, Il a fermé ta robe pour briser tes chaînes.

Devant les roues toutes nouées

Un éventail rit aux éclats.

Dans les traîtres filets de l'herbe

Les routes perdent leur reflet.

Ne peux-tu donc prendre les vagues

Dont les barques sont les amandes

Dans ta paume chaude et câline

Ou dans les boucles de ta tête?

Ne peux-tu prendre les étoiles?

Écartelée, tu leur ressembles,

Dans leur nid de feu tu demeures

Et ton éclat s'en multiplie.

De l'aube bâillonnée un seul cri veut jaillir,

Un soleil tournoyant ruisselle sous l'écorce.

Il ira se fixer sur tes paupières closes.

Ô douce, quand tu dors, la nuit se mêle au jour. 